\title{
A Novel Stochastic Predictive Stabilizer for DC Microgrids Feeding CPLs
}

Kowsari, Elham; Zarei, Jafar; Razavi-Far, Roozbeh; Saif, Mehrdad; Dragicevic, Tomislav; Khooban, Mohammad Hassan

Published in:

IEEE Journal of Emerging and Selected Topics in Power Electronics

Link to article, DOI:

10.1109/JESTPE.2020.3008885

Publication date:

2021

Document Version

Peer reviewed version

Link back to DTU Orbit

Citation (APA):

Kowsari, E., Zarei, J., Razavi-Far, R., Saif, M., Dragicevic, T., \& Khooban, M. H. (2021). A Novel Stochastic Predictive Stabilizer for DC Microgrids Feeding CPLs. IEEE Journal of Emerging and Selected Topics in Power Electronics, 9(2), 1222 - 1232. https://doi.org/10.1109/JESTPE.2020.3008885

\section{General rights}

Copyright and moral rights for the publications made accessible in the public portal are retained by the authors and/or other copyright owners and it is a condition of accessing publications that users recognise and abide by the legal requirements associated with these rights.

- Users may download and print one copy of any publication from the public portal for the purpose of private study or research.

- You may not further distribute the material or use it for any profit-making activity or commercial gain

- You may freely distribute the URL identifying the publication in the public portal

If you believe that this document breaches copyright please contact us providing details, and we will remove access to the work immediately and investigate your claim 


\title{
A Novel Stochastic Predictive Stabilizer for DC Microgrids Feeding CPLs
}

\author{
Elham Kowsari, Jafar Zarei, Member, IEEE, Roozbeh Razavi-Far, Senior Member, IEEE, Mehrdad Saif, Senior \\ Member, IEEE, Tomislav Dragičević, Senior Member, IEEE, Mohammad Hassan Khooban, Senior Member,
} IEEE

\begin{abstract}
In this work, a novel nonlinear approach is proposed for the stabilization of microgrids with constant power loads (CPLs). The proposed method is constructed based on the incorporation of a pseudo-extended Kalman filter into stochastic nonlinear model predictive control (MPC). In order to achieve high-performance and optimal control in DC microgrids, estimating the instantaneous power flow of the uncertain constant power loads and the available power units is essential. Thus, by utilizing the advantages of the stochastic nonlinear model predictive control and the pseudo-extended Kalman filter, an effective control solution for the stabilization of DC islanded microgrids with CPLs is established. This technique develops a constrained controller for practical application to handle the states and control input constraints explicitly; furthermore, as it estimates the current by using the pseudo-EKF, it is a current-senseless approach. As noisy measurements are taken into account for the state estimation, it leads to a less conservative control action rather than the classical robust MPC, whereas it guarantees the global asymptotic stability in the presence of noisy measurements and parameter uncertainty. To validate the performance of the proposed controller, the attained results are compared to state-ofthe-art controllers. Furthermore, the implementability of the proposed method is validated using real-time simulations on dSPACE hardware.
\end{abstract}

Index Terms- DC microgrid, Constant power load, Model-in-the-Loop, Nonlinear dynamic, Stochastic model predictive control, Extended Kalman filter.

\section{INTRODUCTION}

Today's growing energy demand raises various environmental issues such as air pollution, acid rain, water pollution, and the increase of greenhouse gases due to the use of non-renewable energy sources [1], [2]. To overcome these issues, various solutions are suggested. One of the most

E. Kowsari is with the Department of Electrical Engineering, Shiraz University of Technology, Shiraz, Iran (e-mail: e.kosari@ sutech.ac.ir).

J. Zarei is with the Department of Electrical Engineering, Shiraz University of Technology, Shiraz, Iran, and also with the Department of Electrical and Computer Engineering, Windsor University, ON N9B 3P4, Canada. (e-mail: zarei@sutech.ac.ir, jzarei@uwindsor.ca).

R. Razavi-Far and Mehrdad Saif are with the Department of Electrical and Computer Engineering, Windsor University, ON N9B 3P4, Canada. (e-mail: roozbeh@uwindsor.ca, msaif@uwindsor.ca).

T. Dragi 'cevi'c is with the Department of Electrical Engineering, Technical University of Denmark, Elektrovej, 325, 2800 Kgs. Lyngby, Denmark (e-mail: tomdr@elektro.dtu.dk).

M. H. Khooban is with the Department of Engineering, Aarhus University Denmark. (e-mail: mhkhoban@gmail.com, khooban@eng.au.dk). important keys is the use of clean energy sources in modern power grids, which is an appropriate alternative for enhancing the performance and green operation in future power generation grids [3]-[5].

Most industries, companies, and hospitals require a backup source to tolerate uncertainty and perturbation in the distribution network. However, traditional sources such as energy storage systems and diesel generators are known as expensive backups. Therefore, the use of microgrids (MGs) is an affordable and reliable alternative in the energy crisis [6][10]. Moreover, the use of MGs can significantly reduce the loss of energy through the power transmission line.

AC MGs have been used for many years and their functionality and structure is well-known [11]. However, DC MGs possess their specific benefits. The most important of them include: a) DC microgrids are more suitable for connecting to energy storage and renewable resources as they are inherently DC, and b) modern electrical devices such as computers and servers in the data centers require DC power. Even conventional electrical loads are inherently $\mathrm{AC}$; for example, induction motors become a DC load when they are controlled by a variable speed driver. However, in DC microgrids, various ranges of voltage are required. To this aim, electronic power interfaces are used to convert the power. These converters operate as an interface at the various values of voltage. Moreover, the stability issues of these systems are a major concern, especially when they are connected to a constant power load (CPL) in DC modern power grids. Besides, comprehensive studies on CPLs are necessary to address practical concerns in the power distribution systems due to the widespread use of CPLs in DC grids [12], [13]. Moreover, it is well-known that CPLs have negative impedance effects, which may cause instability in a DC microgrid; furthermore, it introduces a destabilizing effect into a DC microgrid that may cause its main bus voltages to show significant oscillations or to collapse. One of the methods that can handle this issue is to connect the energy storage directly to the main bus. Adding energy storage directly connected to the system buses can also eliminate the oscillations caused by the CPL [14].

To overcome these issues, several studies have been carried out [11], [14], [15]. For instance, a linear stabilization approach has been suggested to stabilize DC microgrids (DCMG) [16]. Some works are devoted to adding resistance loads, filters, and energy storages to decrease voltage oscillations [17]. Furthermore, various approaches have been put forward to solve the global asymptotic stability. For instance, in [17] and [19], a novel nonlinear method based on 
feedback linearization has been suggested to guarantee global asymptotic stability. In addition, some approaches based on the sliding mode controller have been presented to stabilize nonlinear DC microgrids [19], [20].

Moreover, as some uncertainties such as measurement noises, modeling errors and uncertainty in the CPLs or source parameters, the exact mathematical model of the DC microgrid cannot be extracted. Hence, the model-based controller designed based on the exact model cannot handle these uncertainties [21], [22]; thus, the performance of the system was degraded. A nonlinear controller that used the sliding-mode duty-ratio controller (SMDC) is considered in the reference [22]. SMDC is able to stabilize the dc power systems over the entire operating range in the presence of significant variations in the load power and input voltage. Sometimes, there are constraints on the currents and voltages of the DC microgrid [23], and a constrained controller should be applied to achieve an acceptable performance. One of the most well-known constrained controllers is the model predictive controller (MPC) in which physical constraints on inputs, outputs, and states can be taken into account [24]. However, some features of the model such as nonlinearity, uncertainty, and measurement noise have not been considered, although they cause the performance of the MPC to degrade. Motivated by the aforementioned issues, the present paper proposes a novel stochastic controller, which has the following advantages compared to a state-of-the-art controller applied to DC microgrids [10]. In this reference, a Kalman based output controller for stabilizing the DCMG with CPLs is developed. The proposed approach employs the cubature Kalman filter, and linear matrix inequality (LMI) to stabilize the DCMG. Sufficient conditions for exponential stability of the robust linear state feedback controller are derived in terms of LMIs. As an advantage, the proposed controller uses the estimated states to stabilize the DCMG, meanwhile, the previous approaches fully utilize all of the states, which is not applicable in practice. Although the presented method addresses the stabilization problem of DCMG while it does not need many sensors to measure the value of all states, it uses a filter and a controller separately to achieve this aim.

Therefore, the time which is taken to calculate the control input at each step might be rather high that results in a slow controller, especially for large dimensional systems. To overcome this disadvantage, in current work, a novel controller is proposed that uses a filter inside the controller. It is an output controller in which all states of the system are not required to calculate the control input while results in a lower computational time rather than the developed approach in reference [10]. Besides, if there are limitations on the voltage and current of each CPL, it can be taken into account to compute the control input.

This work proposes a nonlinear controller that can handle nonlinearity in a DC microgrid. It suggests a new robust controller that shows effective performance in the presence of the measurement noises and model uncertainties of the DC microgrid (DCMG). The proposed novel controller is designed so that it can deal with physical and practical limitations on the current and voltage amplitudes of the DCMG.

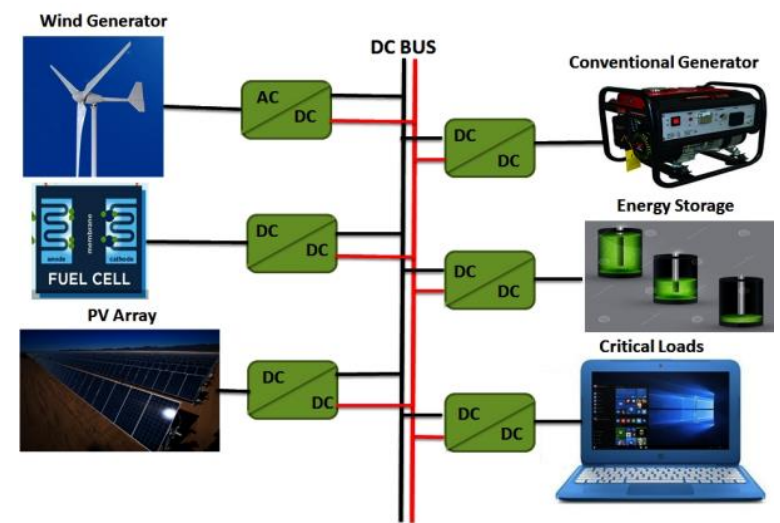

Fig. 1. A simplified schematic of the conventional DC microgrid with various constant power loads.

In addition, for the first time, a theorem is developed that guarantees the global asymptotic stability in the presence of process and measurement noises. To sum up, the highlights of the current study can be summarized as follows:

1- A novel robust stochastic nonlinear MPC is developed based on the pseudo-Kalman filter.

2- Two new theorems are derived to guarantee the global asymptotic stability of the proposed controller and the reliable convergence of EKF algorithms.

3- The simple structure of the proposed control technique facilitates its application for a variety of modern DC power grids with different configurations.

4- To show the performance and robustness of the proposed controller, hardware-in-the-loop (HiL) experiments are conducted.

This paper is organized as follows: the next section examines the dynamics of the DC microgrid under CPLs. The proposed method to guarantee the global asymptotic stabilization of a DC microgrid is presented in the third section. The findings of this paper and real-time simulation results are presented in the fourth section. Finally, the conclusion of the paper is presented in Section $\mathrm{V}$.

\section{CASE STUDY DESCRIPTION}

In this section, the dynamic model of a DC modern power grid is presented. A general schematic of the DC stand-alone power grid is shown in Figure 1 [14].

The dynamic equation of the $i^{\text {th }}$ CPL based on Kirchhoff's circuit laws can be obtained as follows [10]:

$$
\left\{\begin{array}{c}
\dot{I}_{i}=-\frac{R_{i}}{L_{i}} I_{i}-\frac{1}{L_{i}} V_{i}+\frac{1}{L_{i}} V_{s} \\
\dot{V}_{i}=\frac{1}{C_{i}} I_{i}-\frac{1}{C_{i}} \frac{P_{i}}{V_{i}}
\end{array}\right.
$$

where $I_{i}, V_{i}, R_{i}$ and $L_{i}$ are the inductor current, the capacitor voltage, the resistance and inductance of $i^{\text {th }}$ CPL, respectively. This assumes $z_{i}=\left[\begin{array}{ll}I_{i} & V_{i}\end{array}\right]^{T}$ and $z_{s}=\left[\begin{array}{ll}I_{s} & V_{S}\end{array}\right]^{T}$ as the state vector of the $i^{\text {th }} \mathrm{CPL}$ and the state vector of source, respectively. Therefore, the state equation of each CPL can be obtained as follows:

$$
\dot{z}_{i}=A_{i} z_{i}+A_{i s} z_{s}+k_{i} t_{i}
$$




$$
A_{i}=\left[\begin{array}{cc}
-\frac{R_{i}}{L_{i}} & -\frac{1}{L_{i}} \\
\frac{1}{C_{i}} & 0
\end{array}\right], \quad k_{i}=\left[\begin{array}{c}
0 \\
\frac{P_{i}}{C_{i}}
\end{array}\right], \quad A_{i s}=\left[\begin{array}{cc}
0 & \frac{1}{L_{i}} \\
0 & 0
\end{array}\right]
$$

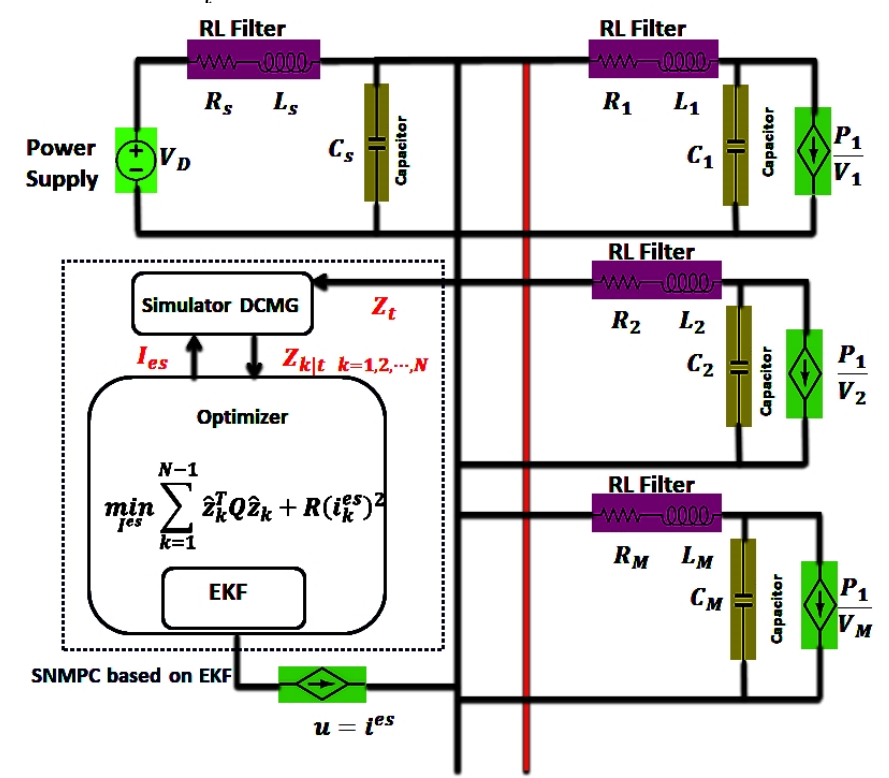

Fig. 2. The DC Microgrid with $M$ constant power loads which is controlled with SNMPC based on EKF.

Due to the existence of the nonlinear term $t_{i}=1 / V_{i}$, the state equation of the CPLs represents nonlinear behavior; therefore, in order to achieve an accurate control mechanism, nonlinear control approaches should be employed.

In the same way, the state equation of the source can be shown as:

$\left\{\begin{array}{c}\dot{I}_{s}=-\frac{R_{s}}{L_{s}} I_{s}-\frac{1}{L_{s}} V_{s}+\frac{1}{L_{s}} V_{D} \\ \dot{V}_{s}=\frac{1}{C_{s}} I_{s}-\frac{1}{C_{s}} V_{s}-\frac{1}{C_{s}} i^{e s}\end{array}\right.$

where $I_{s}, V_{s}, R_{i}$ and $L_{i}$ are the inductor current, the capacitor voltage, the resistance and inductance of the source, respectively, and $V_{D}$ is the power supply. The state-space can be shown as follows:

$$
\begin{gathered}
A_{s}=\left[\begin{array}{cc}
-\frac{R_{s}}{L_{s}} & -\frac{1}{L_{s}} \\
\frac{1}{C_{s}} & 0
\end{array}\right], b_{s}=\left[\begin{array}{c}
\frac{1}{L_{s}} \\
0
\end{array}\right], A_{c n}=\left[\begin{array}{cc}
0 & 0 \\
\frac{1}{C_{s}} & 0
\end{array}\right], \\
b^{e s}=\left[\begin{array}{c}
0 \\
-\frac{1}{C_{s}}
\end{array}\right]
\end{gathered}
$$

By defining an augmented state vector as $Z^{a u g}=$ $\left[\begin{array}{lllll}z_{1}{ }^{T} & z_{2}{ }^{T} & \ldots & z_{M}{ }^{T} & z_{S}{ }^{T}\end{array}\right]^{T}$ the new overall model of the DCMG is shown as

$$
\begin{gathered}
\dot{Z}^{\text {aug }}=A^{a u g} Z^{a u g}+K T+B^{e s} i^{e s}+B_{s} V_{D} \\
\text { where } T=\left[t_{1}, \ldots, t_{M}\right]^{T} \text { and } \\
A^{\text {aug }}=\left[\begin{array}{ccccc}
A_{1} & 0 & \cdots & 0 & A_{1 s} \\
0 & A_{2} & \cdots & 0 & A_{2 s} \\
\vdots & \vdots & \ddots & \vdots & \vdots \\
0 & 0 & \cdots & A_{M} & A_{M s} \\
A_{c n} & A_{c n} & \cdots & A_{c n} & A_{s}
\end{array}\right], B^{e s}=\left[\begin{array}{c}
0 \\
\vdots \\
0 \\
b^{e s}
\end{array}\right],
\end{gathered}
$$

$$
\begin{gathered}
B_{S}=\left[\begin{array}{c}
0 \\
\vdots \\
0 \\
b_{s}
\end{array}\right] \\
K=\left[\begin{array}{cccc}
k_{1} & 0 & \cdots & 0 \\
0 & k_{2} & \cdots & 0 \\
\vdots & \vdots & \ddots & \vdots \\
0 & 0 & \cdots & k_{M} \\
0 & 0 & \cdots & 0
\end{array}\right],
\end{gathered}
$$

As shown in Figure 2, in equation (2), the current injection into the current source is $\mathrm{P}_{\mathrm{i}} / \mathrm{V}_{\mathrm{i}}$, which makes the power injection to be $P_{i}$. Thus, in order to stabilize the DC MG under CPLs, the current $i^{e s}$ is selected as the control input [25]. Now, according to the nonlinear model of the DCMG, a fast and high-performance nonlinear control is necessitated for this case study. Therefore, the next section is provided to present an accurate nonlinear control approach to overcome the difficulties that exist in a DCMG under CPLs.

\section{STOCHASTIC NONLINEAR MODEL PREDICTIVE CONTROLLER BASED ON PSEUDO-EXTENDED KALMAN FILTER}

In this section, a new constrained control approach is developed to control a DCMG with CPLs. The proposed approach is constructed based on a nonlinear model predictive control (NMPC), which is a well-known approach to control nonlinear systems in the presence of state and control input constraints [26], [27]. Traditional NMPC techniques assume that the states of the system are deterministic and there are no uncertainty and errors in the measurements; therefore, the cost function is described as

$$
J\left(z_{k}, \boldsymbol{u}\right)=\sum_{k=1}^{N-1} z_{k}^{T} Q z_{k}+u_{k}^{T} R u_{k}+z_{N}^{T} P z_{N}
$$

where $z_{k}$ and $z_{N}$ are deterministic states and $u=$ $\left\{u_{1}, u_{2}, \cdots, u_{N-1}\right\}$ is the sequence of the control input during the horizon time $N$.

However, in practice, there are some uncertainties in the dynamic of these systems. The system uncertainty is generally categorized as a) the uncertain model structure or parameters, b) the uncertain initial condition, or c) the unmeasurable exogenous disturbance. The most common form of the system, which includes the uncertainty in the dynamic is represented as follows:

$$
\begin{gathered}
z_{k+1}=f\left(z_{k}, u_{k}\right)+w_{k} \\
y_{k}=g\left(z_{k}, u_{k}\right)+v_{k}
\end{gathered}
$$

where $z_{k} \in \mathbb{R}^{n_{z}}$ denotes the state of the system, $y_{k} \in \mathbb{R}^{n_{y}}$, and $u_{k} \in \mathbb{R}^{n_{u}}$ are the measurement and control inputs, respectively. It is worth noting that $w_{k}$ can be interpreted as the term that reflects the effect of modeling uncertainty or exogenous disturbance on the state of the system. Moreover, $w_{k}$ and $v_{k}$ are the sequences of independent and identically distributed (i.i.d.) variables that denote the system disturbance and measurement noise, respectively. In addition, $E\left(w_{k} v_{k}^{T}\right)=$ $0, E\left(w_{k} w_{k}^{T}\right)=Q_{k}$ and $E\left(v_{k} v_{k}^{T}\right)=R_{k}$. As the model of the system has stochastic terms, the formulation of the NMPC should be changed such that the stochastic nature of the system is considered. Therefore, Eq. (7) should be also described based on the stochastic terms. In this paper, the 
expected value of the cost function, $E\left(J\left(z_{k}, \boldsymbol{u}\right)\right)$, is applied to show the stochastic essence of the system. Thus, the cost function has been modified. To this aim, let us assume the difference between the true state and its mean as $e_{k}=z_{k}-$ $\hat{z}_{k}$, in which $\hat{z}_{k}=E\left(z_{k}\right)$.

Substituting $e_{k}$ to the Eq. (7), yields:

$$
\begin{aligned}
E\left(J\left(z_{k}, \boldsymbol{u}\right)\right)=E & \left(\sum_{k=1}^{N-1} z_{k}^{T} Q z_{k}+2 z_{k}^{T} Q e_{k}+e_{k}^{T} Q e_{k}\right. \\
& +u_{k}^{T} R u_{k}+z_{N}^{T} P z_{N}+2 z_{N}^{T} P e_{N} \\
& \left.+e_{N}^{T} P e_{N}\right)+\Pi
\end{aligned}
$$

where $\Pi$ denotes $E\left(\sum_{k=1}^{N-1} e_{k}^{T} Q e_{k}+e_{N}^{T} P e_{N}\right)$ that does not comprise the control input; therefore, it can be removed from the optimization problem. As $e_{k}$ has a zero mean, and it is uncorrelated from the state, the cost function is obtained as follows:

$$
E\left(J\left(z_{k}, \boldsymbol{u}\right)\right)=\sum_{k=1}^{N-1} \hat{z}_{k}^{T} Q \hat{z}_{k}+u_{k}^{T} R u_{k}+\hat{z}_{N}^{T} P \hat{z}_{N}
$$

Furthermore, to reflect the stochastic concept of the state, the constraints should be replaced with appropriate equalities and inequalities. To this aim, two methods are proposed. The first method uses the expected value of the constraints. For instance, the state constraint $G z_{k} \leq h$ can be replaced with $E\left(G z_{k}\right) \leq h$. The second method uses the chance constraints of the states in which the probability constraint is shown as follows:

$$
P_{k}\left[G_{j} z_{k} \leq h_{j}\right] \geq 1-\alpha_{j}, \quad j=0,1, \cdots, n_{s}
$$

where $\alpha_{j}$ is a predetermined term, which is shown by the allowed probability of violation of the state constraint. Note that Eq. (11) cannot be used directly in the optimization problem; thus, a suitable alternative inequality that can be easily utilized in the optimization problem is needed. To this end, [28] used inscribed conic sets that constraint Eq. (11) can be given by the following constraints:

$$
\begin{gathered}
\varphi^{-1}\left(1-\alpha_{j}\right) \sqrt{h_{j}^{(i) T} \Sigma_{z}(n+j \mid n) h_{j}^{(i)}}+h_{j}^{(i) T} \hat{x}(n+j \mid n) \\
\leq g_{j}^{(i)}, \quad i=1, \cdots, n_{g_{j}}
\end{gathered}
$$

where $\varphi^{-1}($.$) is the quantile function of the standard Gaussian$ probability distribution. $n_{g_{j}}$ denotes the number of rows of $g_{j}$, $h_{j}^{(i)}$ is the $i^{t h}$ row of the $H_{j}$ and finally $g_{j}$ is the $i^{\text {th }}$ element of $g$.

To sum up, the stochastic model predictive control is formulated as follows:

$$
\begin{gathered}
\min _{\boldsymbol{u}} \sum_{k=1}^{N-1} \hat{z}_{k}^{T} Q \hat{z}_{k}+u_{k}^{T} R u_{k}+\hat{z}_{N}^{T} P \hat{z}_{N} \\
s t: z_{k+i+1 \mid t}=f\left(z_{k+i \mid t}, u_{k+i \mid t}\right)+w_{k+i} \\
y_{k}=g\left(z_{k}, u_{k}\right)+v_{k} \\
u_{k+i \mid t} \in U, k=t, t+1, \cdots, N-1 \\
P_{k}\left[G_{j} z_{k+i+1} \leq h_{j}\right] \geq \alpha_{j} \\
z_{k \mid t}=x_{t \mid t}
\end{gathered}
$$

As seen in Eq. (13), the state estimation is needed to solve the optimization problem in the stochastic systems. It is required a method to find $\hat{z}_{k}$ from an initial time $t$ up to time $k=N$. Several approaches have been developed to calculate state estimation.

Table I. EKF Algorithm

1.Initialization:

2. for $k=1,2, \cdots$

$$
\begin{aligned}
& z_{0}^{+}=E\left(z_{0}\right) \\
& \Sigma_{0}^{-}=E\left[\left(z_{0}-z_{0}^{+}\right)\left(z_{0}-z_{0}^{+}\right)^{T}\right]
\end{aligned}
$$

a) Compute the partial derivation matrix of the system

$$
\boldsymbol{F}_{\boldsymbol{k}}=\left.\frac{\partial f\left(\hat{\mathbf{z}}_{\boldsymbol{k}}, \boldsymbol{i}^{e s}{ }_{k}\right)}{\partial z}\right|_{z_{k}^{+}}
$$

b) Perform the time update for the state estimation and error covariance

$$
\begin{aligned}
& \hat{\boldsymbol{z}}_{k}^{-}=\hat{\boldsymbol{z}}_{k-1}+T_{s} f\left(\hat{\boldsymbol{z}}_{k-1}, u_{k-1}\right) \\
& \Sigma_{k}^{-}=F_{k-1} \Sigma_{k-1} F_{k-1}^{T}+Q_{k-1}
\end{aligned}
$$

c) Compute the partial derivation matrix of the measurement equation

$$
\boldsymbol{H}_{\boldsymbol{k}}=\left.\frac{\partial g\left(\hat{\boldsymbol{z}}_{\boldsymbol{k}}, \boldsymbol{u}_{\boldsymbol{k}-1}\right)}{\partial z}\right|_{z_{\boldsymbol{k}}^{-}}
$$

d) Perform the time update for the state estimation and error covariance

$$
\begin{aligned}
K_{k} & =\Sigma_{k}^{-} H_{k}^{T}\left(H_{k} \Sigma_{k}^{-} H_{k}^{T}+R_{k}\right)^{-1} \\
\hat{\mathbf{z}}_{k} & =\hat{\boldsymbol{z}}_{k}^{-}+K_{k}\left(y_{k}-H_{k} \hat{\mathbf{z}}_{k}^{-}\right) \\
\boldsymbol{\Sigma}_{k} & =\left(I-K_{k} H_{k}\right) \Sigma_{k}^{-}
\end{aligned}
$$

As the model of the system is nonlinear, in this paper, extended Kalman filter (EKF) is exploited for the state estimation [29]-[33] to solve the optimization problem.

The proposed method is called stochastic nonlinear model predictive control (SNMPC) abridged based on pseudo-EKF. The algorithm of the EKF is shown in Table I. In order to use the EKF in the optimization problem, it is supposed that it is divided into two parts. The first part, i.e., time update, is applied to calculate the state estimation for the nonlinear optimization problem in the SNMPC, where it runs $N$ step. The second part, i.e., measurement update, is used to produce the mean and covariance for the next time step.

According to Table I, the implementation of the SNMPC based-on pseudo-EKF for the DC microgrid is shown as follows:

$$
\begin{gathered}
\min _{I^{e s}} \sum_{k=1}^{N-1} \hat{z}_{k}^{T} Q \hat{z}_{k}+R\left(i_{k}^{e s}\right)^{2}+l\left(\hat{z}_{N+i \mid i}\right) \\
s t: \hat{z}_{k+i+1 \mid t}^{-}=\hat{z}_{k+i \mid t}+T_{s} f\left(\hat{z}_{k+i \mid t}, i_{k+i \mid t}^{e s}\right) \\
\Sigma_{k+i+1 \mid t}^{-}=F_{k+i \mid t} \Sigma_{k+i \mid t} F_{k+i \mid t}^{T}+Q_{k+i \mid t} \\
P_{k}\left[G_{j} z_{k+i+1} \leq h_{j}\right] \geq \alpha_{j} \\
u_{k+i \mid t} \in U, \quad t, t+1, \cdots, N-1 \\
z_{k \mid t}=\hat{z}_{k \mid t} \\
z_{N} \in Z_{f} \\
K_{k}=\Sigma_{k}^{-} H_{k}^{T}\left(H_{k} \Sigma_{k}^{-} H_{k}^{T}+R_{k}\right)^{-1} \\
\hat{z}_{k}=\hat{z}_{k}^{-}+K_{k}\left(y_{k}-H_{k} \hat{z}_{k}^{-}\right) \\
\Sigma_{k}=\left(I-K_{k} H_{k}\right) \Sigma_{k}^{-}
\end{gathered}
$$

where $\boldsymbol{I}^{e s}=\left\{i_{1}^{e s}, i_{2}^{e s}, \cdots, i_{N-1}^{e s}\right\}$ is the sequence of the control input during the horizon time, and $l\left(\hat{z}_{N+i \mid i}\right)=\hat{z}_{N+i \mid i}^{T} P \hat{z}_{N+i \mid i} . \Sigma_{k}$ is the covariance matrix at the sample instance $k$.

The stability analysis is the most important issue in designing an efficient controller. Therefore, Theorem 1 is driven to prove the stability of the proposed approach. 
Assumption 1: The terminal set $Z_{f}$ is a positive invariant set under the local control law $v\left(z_{N}\right), \quad 0 \in Z_{f}$, and $f\left(\hat{z}_{k+i \mid i}, v\left(z_{k+i}\right)\right) \in Z_{f}, \quad \forall z \in Z_{f}$.

Assumption 2: Terminal cost $\hat{z}_{N}^{T} P \hat{Z}_{N}$ is a Lyapunov function inside the set $Z_{f}$, therefore, it satisfies $l\left(z_{N+i+1 \mid i}\right)-l\left(z_{N+i \mid i}\right) \leq$ $-\left(\hat{z}_{k+i \mid i}^{* T} Q \hat{z}_{k+i \mid i}^{*}+R\left(v\left(z_{i+k \mid i}\right)\right)^{2}\right), \forall z_{i+k} \in Z_{f}$ for all $\hat{z} \in$ $Z_{f}$, where $\hat{z}^{*}{ }_{k}$ and $i_{k}^{e s *}$ are the optimal value of the state and the control input.

Theorem 1. Under Assumptions (1) and (2), the closed-loop system controlled by the SNMPC based on the pseudo-EKF control law is asymptotically stable if the EKF algorithm is convergent.

Proof: By assuming that EKF is convergent, which will be proven in the next theorem, firstly, we need to show the recursive feasibility of the controller. It assumes that $\hat{z}_{k}$ is feasible and its corresponding optimal control input sequence is $\left\{i_{0}^{e s *}, i_{2}^{e s *}, \cdots i_{N-2}^{e s *}\right\}$. Based on the assumption 1, the sequence $\left\{i_{1}^{e s *}, i_{2}^{e s *}, \cdots i_{N-1}^{e s *}\right\}$ is also feasible for $\hat{z}_{k+1}$. Therefore, the proposed controller is recursive feasible, if it starts at a feasible initial state $\hat{z}_{k}$.

Then, to illustrate the asymptotic stability, it is necessary to show that the cost function is decreasing during the time. To this aim, as the optimal cost function can be a candidate for the Lyapunov function, we just need to show that the following inequality is satisfied:

$V^{*}\left(\hat{z}_{i+1}\right)-V^{*}\left(\hat{z}_{i}\right) \leq 0 \quad \forall \hat{z}_{k} \neq 0$

where $V^{*}\left(\hat{z}_{i}\right)=\sum_{k=1}^{N-1} \hat{z}_{k+i \mid i}^{* T} Q \hat{z}_{k+i \mid i}^{*}+R\left(i_{k+i \mid i}^{e s *}\right)^{2}+l\left(z_{N+i \mid i}\right)$ is the optimal cost function for the optimal sequence of $\tilde{I}_{i}^{e s *}=$ $\left\{i_{1}^{e s *}, i_{2}^{e s *}, \cdots i_{N-1}^{e s *}\right\}$.

The sub-optimal cost function for the sub-optimal control sequence $\tilde{I}_{i+1}^{e s}=\left\{i_{i+1}^{e s *}, i_{i+2}^{e s *}, \cdots i_{i+N-1}^{e s *}, v\left(z_{i+N \mid}\right)\right\}$ for $\hat{z}_{i+1}$ is as follows:

$$
\begin{gathered}
\hat{V}\left(\hat{z}_{i+1}\right)= \\
\sum_{k=1}^{N} \hat{z}_{k+i \mid i}^{T} Q \hat{z}_{k+i \mid i}+R\left(i_{k+i \mid i}^{e s *}\right)^{2}+l\left(f\left(\hat{z}_{N+i}, v\left(z_{N+i \mid i}\right)\right)\right)
\end{gathered}
$$

adding and subtracting $l\left(\hat{z}_{N+i \mid i}\right)$ to Eq. (16):

$$
\begin{aligned}
& \overbrace{\sum_{k=0}^{N-1} \hat{z}_{k+i \mid i}^{T} Q \hat{z}_{k+i \mid i}+R\left(i_{k+i \mid i}^{e s}\right)^{2}+l\left(\hat{z}_{N+i \mid i}\right)}^{V^{*}\left(\hat{z}_{i}\right)}- \\
& l\left(\hat{z}_{N+i \mid i}\right)-\left(\hat{z}_{i \mid i}^{T} Q \hat{z}_{i \mid i}+R\left(i_{i \mid i}^{e s *}\right)^{2}\right)-\left(\hat{z}_{i+N \mid i}^{T} Q \hat{z}_{N+i \mid i}+\right. \\
& \left.R\left(i_{i+N \mid i}^{e s *}\right)^{2}\right)+l\left(f\left(\hat{z}_{N+i}, v\left(z_{N+i \mid i}\right)\right)\right) \\
& \text { Since } V^{*}\left(\hat{z}_{i+1}\right) \leq \hat{V}\left(\hat{z}_{i+1}\right) \\
& V^{*}\left(\hat{z}_{i+1}\right) \leq V^{*}\left(\hat{z}_{i}\right)-l\left(\hat{z}_{N+i \mid i}\right)-\left(\hat{z}_{i \mid i}^{T} Q \hat{z}_{i \mid i}+\right. \\
& \left.R\left(i_{i \mid i}^{e s *}\right)^{2}\right)-\left(\hat{z}_{i+N \mid i}^{T} Q \hat{z}_{N+i \mid i}+R\left(i_{i+N \mid i}^{e s *}\right)^{2}\right)+ \\
& l\left(f\left(\hat{z}_{N+i}, v\left(z_{N+i \mid i}\right)\right)\right) \\
& \text { with Assumption } 2: \\
& -l\left(\hat{z}_{N+i \mid i}\right)-\left(\hat{z}_{i+N \mid i}^{T} Q \hat{z}_{N+i \mid i}+R\left(i_{i+N \mid i}^{e s *}\right)^{2}\right)+
\end{aligned}
$$

$l\left(f\left(\hat{z}_{N+i}, v\left(z_{N+i \mid i}\right)\right)\right) \leq 0$

therefore,

$$
\begin{gathered}
V^{*}\left(\hat{z}_{i+1}\right)-V^{*}\left(\hat{z}_{i}\right) \leq-\left(\hat{z}_{i \mid i}^{T} Q \hat{z}_{i \mid i}+R\left(i_{i \mid i}^{e s *}\right)^{2}\right) \\
V^{*}\left(\hat{z}_{i+1}\right)-V^{*}\left(\hat{z}_{i}\right)<0 \quad \forall \hat{z}_{i} \neq 0
\end{gathered}
$$

To show the convergence of the EKF, Theorem 2 and its proof are presented. To this aim, consider the following assumptions and Lemma 1.

Assumption 3: There are for all $k>0, f, h, p_{1}, p_{2}, q, r>0$ such that the following conditions hold:

$$
\begin{gathered}
\left\|F_{k}\right\| \leq f,\left\|H_{k}\right\| \leq h, \\
\sigma_{1} I \leq \Sigma_{k} \leq \sigma_{2} I, q I \leq Q_{k}, r I \leq R_{k}
\end{gathered}
$$

Assumption 4: for $\forall k>0, F$ is a non-singular matrix.

Lemma 1: Based on Assumptions 3 and 4, there is $0 \leq \beta \leq 1$ so that:

$$
\left(I_{k}-K_{k} H_{k}\right)^{T} F_{k}^{T} \Sigma_{k+1}^{-1} F_{k}\left(I-K_{k} H_{k}\right) \leq \Sigma_{k}^{-1}(1-\beta)
$$

\section{Proof of lemma 1:}

Based on the one-step EKF algorithm [34], we have

$$
\Sigma_{k+1}=F_{k} \Sigma_{k} F_{k}^{T}+Q_{k}-F_{k} \Sigma_{k} H_{k}^{T} \Sigma_{k}^{T} F_{k}^{T}
$$

which can be rewritten as follows:

$$
\begin{aligned}
& \Sigma_{k+1}=F_{k}\left(I-K_{k} H_{k}\right) \Sigma_{k}\left(I-K_{k} H_{k}\right)^{T} F_{k}^{T}+Q_{k} \\
& +F_{k} K_{k} H_{k} \Sigma_{k}\left(I-K_{k} H_{k}\right)^{T} F_{k}^{T}
\end{aligned}
$$

The last term in the above equation is positive definite, therefore, the following inequality holds:

$$
\Sigma_{k+1} \geq F_{k}\left(I-K_{k} H_{k}\right) \Sigma_{k}\left(I-K_{k} H_{k}\right)^{T} F_{k}^{T}+Q_{k}
$$

by using the matrix inversion lemma, we have:

$$
\begin{aligned}
& \Sigma_{k+1} \geq\left(F_{k}-F_{k} K_{k} H_{k}\right) \\
& {\left[\Sigma_{k}+\left(F_{k}-F_{k} K_{k} H_{k}\right)^{-1} F_{k}^{T}+Q_{k}\left(F_{k}-F_{k} K_{k} H_{k}\right)^{-T}\right]} \\
& \left(F_{k}-F_{k} K_{k} H_{k}\right)^{-T}
\end{aligned}
$$

then, by using Assumption 3:

$$
\begin{aligned}
& \quad \Sigma_{k+1} \geq \\
& \quad F_{k}\left(I-K_{k} H_{k}\right)\left[\Sigma_{k}+\frac{q}{\left(f+f \sigma_{2} h^{2} / r\right)^{2}} I\right]\left(I_{k}-K_{k} H_{k}\right)^{T} F_{k}^{T} \\
& \text { by taking inverses of both sides and multiplying from left by } \\
& F_{k}\left(I-K_{k} H_{k}\right) \text { and from the right by } F_{k}\left(I-K_{k} H_{k}\right)^{T} F_{k}^{T} \text { : } \\
& \qquad \begin{array}{l}
\left(I_{k}-K_{k} H_{k}\right)^{T} F_{k}^{T} \Sigma_{k+1}^{-1} F_{k}\left(I-K_{k} H_{k}\right) \\
\leq \Sigma_{k}^{-1}\left[I+\frac{q}{\sigma_{2}\left(f+f \sigma_{2} h^{2} / r\right)^{2}} I\right]^{-1}
\end{array}
\end{aligned}
$$

by taking inverses of both sides and multiplying from left by

By assuming that:

$$
1-\beta=\left[I+\frac{q}{\sigma_{2}\left(f+f \sigma_{2} h^{2} / r\right)^{2}} I\right]^{-1}
$$

Theorem 2: The EKF algorithm is asymptotically convergent if $v_{k+1}-v_{k}<-\alpha\left(e_{k}\right)$, which $\alpha($.) is a positive definite function and $v_{k}=e_{k}^{T} P_{k}^{-1} e_{k}$.

Proof: To show the convergence of EKF, let us define $e_{k}=$ $z_{k}-\hat{z}_{k}$. By substituting $\hat{z}_{k}$ from the EKF algorithm, the dynamic of $e_{k+1}$ is calculated as follows:

$e_{k+1}=F_{k}\left(I-K_{k} H_{k}\right) e_{k}$

To prove the stability of the EKF, it needs to show that the Lyapunov candidate $v_{k}=e_{k}^{T} \Sigma_{k}^{-1} e_{k}$ is decreasing. By using 


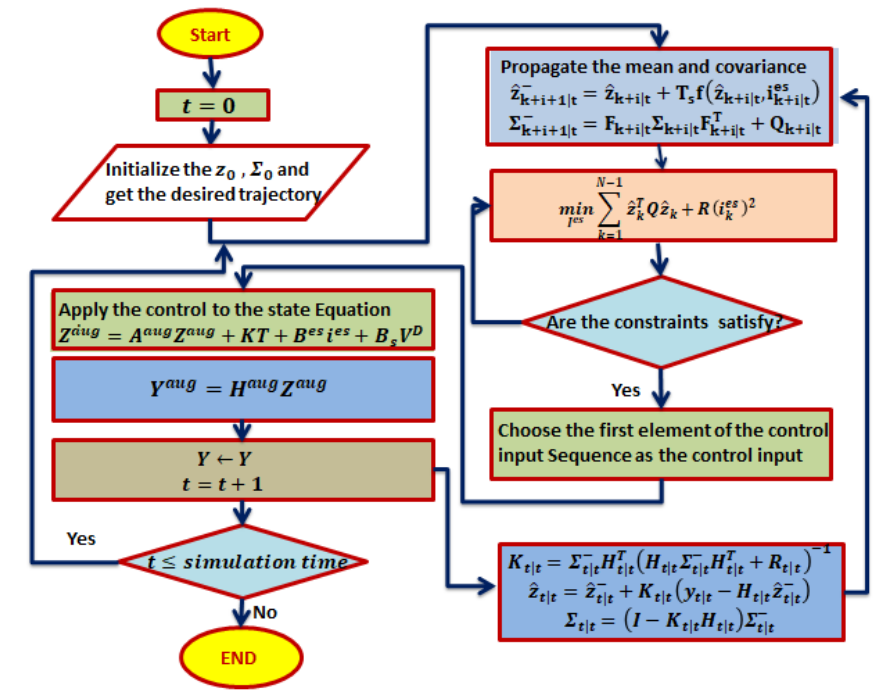

Fig. 3. The flowchart of the DC microgrid control under CPL by using the stochastic nonlinear model predictive control based on EKF.

the result of Lemma 1 and pre and post multiplying by $e_{k}^{T}$, respectively, we have:

$$
\begin{aligned}
& \underbrace{e_{k}^{T}\left(I_{k}-K_{k} H_{k}\right)^{T} F_{k}^{T} \Sigma_{k+1}^{-1} F_{k}\left(I-K_{k} H_{k}\right) e_{k}}_{V_{k+1}} \\
& \leq e_{k}^{T} \Sigma_{k}^{-1}(1-\beta) e_{k}=\underbrace{e_{k}^{T} \Sigma_{k}^{-1} e_{k}}_{v_{k}}-e_{k}^{T} \Sigma_{k}^{-1} \beta e_{k} \\
& v_{k+1}-v_{k} \leq-e_{k}^{T} \Sigma_{k}^{-1} \beta e_{k}=\alpha\left(e_{k}\right)
\end{aligned}
$$

The details of implementing the proposed method for the control of the DC microgrid is shown as a flowchart in Figure 3.

To sum up, the SNMPC based on the EKF is proposed, and its formulation is presented in this section. To show its global asymptotic stability, the Theorem 1 has been derived. Following, three scenarios are studied to evaluate the performance of the DC microgrid in different situations.

\section{REAL-TIME SIMULATION RESULTS}

The main aim of this section is to evaluate the implementability of the SNMPC based on the pseudo-EKF for a DC islanded modern grid under CPL by using Model-in-theLoop (MiL) real-time simulations on dSPACE emulator. Moreover, different scenarios are taken into account to evaluate its practical implementation issues. Several possible situations for the system are considered, and the computational complexity of the proposed method using dSPACE is investigated. It is worth noting that the validity of the applied model is confirmed based on the experimental results obtained in [35]-[38], in which to evaluate the implementation validity of the DC microgrids under CPLs, experimental tests and hardware-in-the-loop real-time simulation are conducted on the same model [36]. Accordingly, as the validity of the applied model is justified, MiL simulations results confirm that the computational complexity of the proposed controller is feasible in practice, and the interactions between the controller and the system comply with the system requirements.
Remark 1: The control input of the proposed NMPC is derived by solving a nonlinear programming problem (NLP). However, most NLP solvers suffer from a high computational complexity when the numbers of states and input constraints are high. To overcome this issue, the CasADi toolbox has been used to solve the optimization problem with a large degree of flexibility [39]. The applicability of the proposed NMPC in the aspect of computational complexity is verified through MiL simulations, in which the CasADi toolbox is utilized to solve the NLP. In other words, by utilizing this toolbox that is significantly faster than the fmincon function in MATLAB, the computational complexity is decreased to guarantee the feasibility of the algorithm in practice.

To show the efficiency and performance of the novel proposed scheme in the control of DC microgrids, three different scenarios are investigated. In this study, the DC microgrid feeding CPLs is considered and the parameters are reported in Table II.

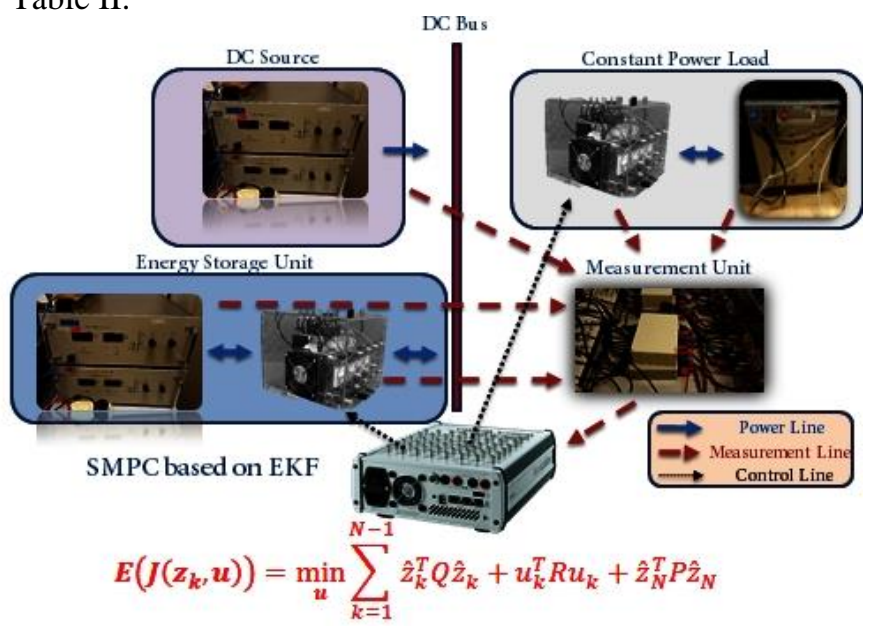

Fig. 4. The schematic of the real-time controller.

As the DC microgrid is characterized by a continuous dynamic, the Euler method, with the sampling time $T_{s}=$ $100 \mu s$ is employed to discretize the model, and the switching rate of pulse duration modulation (PWM) is $20 \mathrm{KHz}$. The tuning parameters of the proposed controller are $\mathrm{N}$ (the prediction horizon), and the weighted matrices ( $Q$ and $R$ ) and $Q_{k}$ and $R_{k}$ which are chosen as $\mathrm{N}=2, \mathrm{Q}=\mathrm{I}_{4}, \mathrm{R}=1, \mathrm{Q}_{\mathrm{k}}=$ $10^{-2} \mathrm{I}_{4}$ and $\mathrm{R}_{\mathrm{k}}=10^{-4}$. The state weighting matrix $\mathrm{Q}$ in the cost function of the proposed controller allows the states to be weighed according to their relative importance. Similarly, R allows input to be weighted according to their relative importance. In other words, the most important variable has the largest weight-input, and increasing the values of weights tends to make the SMPC more conservative by reducing the magnitudes of the control input. $Q_{k}$ and $R_{k}$ are the tuning parameters that adjust the performance of pseudo-EKF, and they are chosen based on each application. The value of $Q_{k}$ and $\mathrm{R}_{\mathrm{k}}$ determine the uncertainty of the process model and the measurement, respectively.

The MiL real-time simulation technique-based on dSPACE 1202 board has been chosen as the rapid prototyping solution to verify the fulfillment of the developed method. The overall structure for verifying the proposed SNMPC based on the pseudo-EKF is depicted in Figure 4. 
Table II. DCMG and CPL Parameters

\begin{tabular}{ccc}
\hline \hline$R_{1}=1.1 \Omega$ & $R_{s}=1.1 \Omega$ & $L_{s}=39.5 \mathrm{mH}$ \\
$C_{1}=500 \mu \mathrm{F}$ & $C_{s}=500 \mu \mathrm{F}$ & $L_{1}=39.5 \mathrm{mH}$ \\
$P_{1}=300 \mathrm{~W}$ & $V_{D}=200 \mathrm{~V}$ & \\
Power rate of energy & SEMIKRON converter & \\
storage: Max 600WT & & \\
\hline \hline
\end{tabular}

\section{A. Scenariol:}

In this section, to show the advantages of the SNMPC based on the pseudo-EKF, firstly, it is compared to the conventional NMPC and the attainted results are presented in Figure 5. As shown in this figure, there are measurement noises and resistance variations in the DC microgrid where the conventional NMPC strictly depends on the accurate model of the DC microgrid, therefore, the conventional controller fails to regulate the states of the system to the acceptable values. Furthermore, three common error measurement criteria include the sum of squared errors (SSE), mean absolute error (MAE), and mean square error (MSE), which are used to evaluate the performance of the SNMPC based on the pseudoEKF and the traditional NMPC. The SSE essentially measures the variation of tracking errors. Generally, a lower SSE indicates that the controller can track better the desired value explains the data while a higher SSE indicates that the poorly tracking the desired value explains the data. The SSE can be calculated by $\mathrm{SSE}=\sum_{\mathrm{i}=1}^{\mathrm{n}}\left(\mathrm{x}_{\mathrm{i}}-\mathrm{x}_{\mathrm{ref}}\right)$. Moreover, MAE and MSE are two of the most common metrics used to measure accuracy. MAE measures the average magnitude of the errors in a set of predictions, without considering their direction. It is the average over the test sample of the absolute differences between desired value and control value where all individual differences have equal weight, $\mathrm{MAE}=\frac{1}{\mathrm{n}} \sum_{\mathrm{i}=1}^{\mathrm{n}}\left|\mathrm{x}_{\mathrm{i}}-\mathrm{x}_{\mathrm{i}, \mathrm{ref}}\right|$. MSE is a quadratic scoring rule that measures the average magnitude of the error. It is the square root of the average of squared differences between desired value and control value, $\operatorname{MSE}=\frac{1}{n} \sum_{i=1}^{n}\left(x_{i}-x_{\text {ref }}\right)^{2}$. As shown in Figure 6 , these criteria are close to zero in the proposed method, which shows the superiority of the proposed method.

Secondly, to assess the performance of the SNMPC based on the pseudo-EKF, it is compared with two different methods. The first one is the proportional-integral (PI) controller and the second one is the proposed approach in reference [10]. The initial condition is chosen as $z_{0}=[1.4,195,1.4,196]^{T}$ and the covariance matrix is set to $\Sigma_{0}=$ $\operatorname{diag}\left(\left[10^{-2}, 10^{2}, 10^{-2}, 10^{2}\right]\right)$. The simulation results are depicted in Figure 7. This test demonstrates that the SNMPC based on the pseudo-EKF is the fastest method in reaching the operating point.

Furthermore, by using the proposed method, the voltage oscillations are the least in comparison with other methods. Furthermore, the proposed method can deal with states and control input constraints. Conversely, the SNMPC based on the pseudo-EKF will be a suitable choice in which there are bounds on the voltage or current of the DC microgrid or energy storage.

As shown in Figure 7, the driving $i_{e s}$ from the SNMPC-based pseudo-EKF reacts with low oscillation compared to the other controllers. Furthermore, the SNMPC based on the EKF achieves satisfactory performance in the short transient response, and as the states of the DC microgrid represent low oscillations, they can quickly converge with their references.

To sum up, PI is a linear controller, and its performance depends greatly on the operating points of the DC microgrid. Thus, it is not surprising that it shows poor performance in the control of nonlinear systems, due to the linearization errors. However, the proposed method in reference [10] is a nonlinear controller, which results in more oscillations to reach the operating points than other approaches. Furthermore, it cannot handle constraints on the load current and voltage.

\section{B. Scenario 2: Robustness of the SNMPC based on the pseudo-EKF against parameter uncertainty}

In this scenario, to show the robustness of the proposed controller against resistance changes, assume that the resistance of the load is changing over time as follows:

$$
R_{1}=\left\{\begin{array}{lll}
R_{1} & \text { if } & t \leq 0.5 \\
R_{1}+0.2 R_{1} & \text { if } & t>0.5
\end{array}\right.
$$

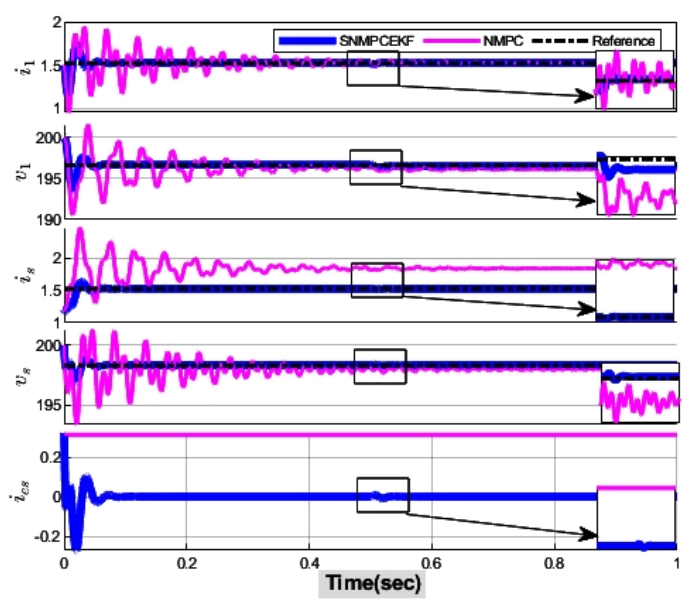

Fig. 5. SNMPC based on pseudo-EKF compared to NMPC (Simulation results).

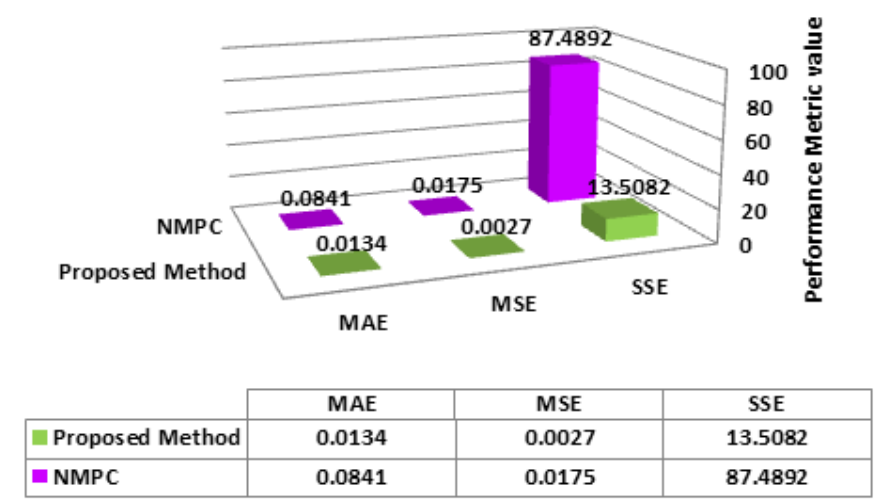

Fig. 6. The comparison of the proposed method with the NMPC.

As shown in Figure 8, although the resistance value changes at $t=5 \mathrm{~s}$, the proposed controller can effectively deal with this change and force the system to return to its operational point. Furthermore, after a short time, the inductance plays a role as a short circuit and the capacitance plays a role as an open 
circuit; therefore, inductance and capacitance variations have a negligible effect in the steady-state.

To verify the robustness of the proposed controller inductance and capacitance variations, the change of the capacitance of the CPL is considered as follows:

$$
C_{1}= \begin{cases}C_{1} & \text { if } \quad t \leq 0.5 \\ 10 * C_{1} & \text { if } t>0.5\end{cases}
$$

The attained results are shown in Figure 9. As shown in this figure, the effect of the capacitance variations is overwhelmed in the voltage and current of the DC microgrid; therefore, it can be concluded that the proposed controller is robust against all kinds of uncertainties, such as resistance, capacitance, and inductance variations.

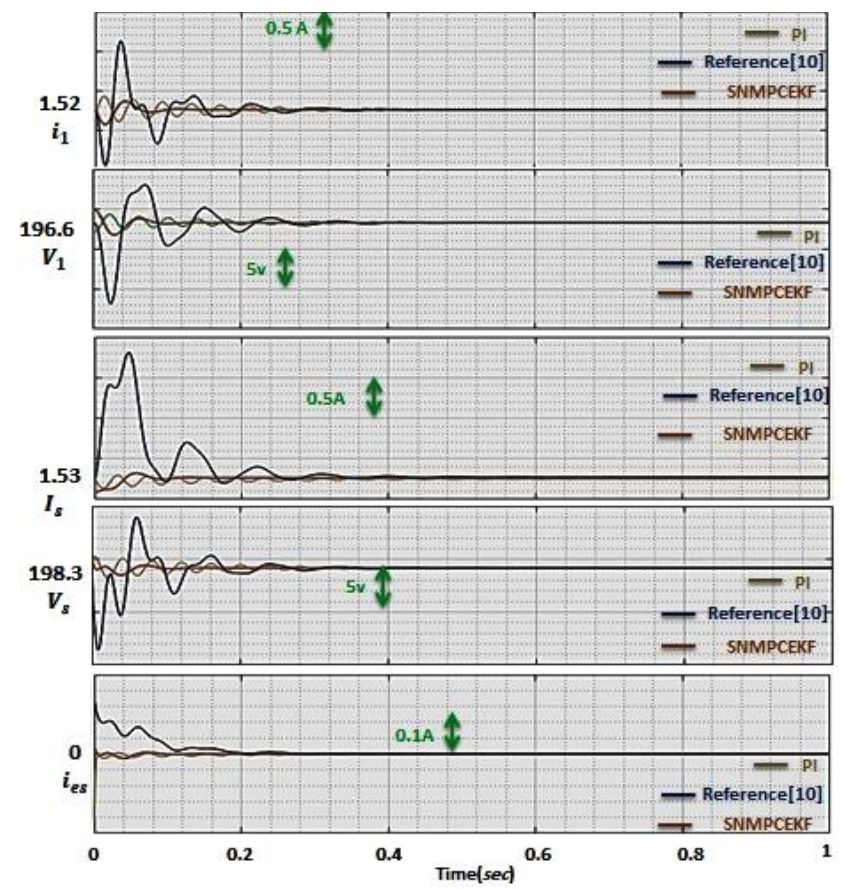

Fig. 7. The comparison of SNMPC based on pseudo-EKF with the PI controller and reference [10] (Real-time simulation).

For the sake of brevity, the result of using different inductances, which is the same as capacitance variations, is not presented.

C. Scenario 3: performance of the SNMPC based on the pseudo-EKF against CPL, input voltage and reference voltage changes

In this scenario, assume that the power of the CPL overtime changes as follows:

$$
P_{1}=\left\{\begin{array}{cr}
300 & t \leq 0.5 \\
350 & 0.5<t<0.7 \\
100 & 0.7<t<1
\end{array}\right.
$$

Furthermore, to verify the performance of the SNMPC based on the pseudo-EKF against the input voltage changes, the voltage variation is assumed as follows:

$$
V_{D}=\left\{\begin{array}{cr}
200 & t \leq 0.5 \\
180 & 0.5<t<0.7 \\
200 & 0.7<t<1
\end{array}\right.
$$

As shown in Figures 10 and 11, the proposed method can track the variations of the load in the DC microgrid with the lowest oscillations.

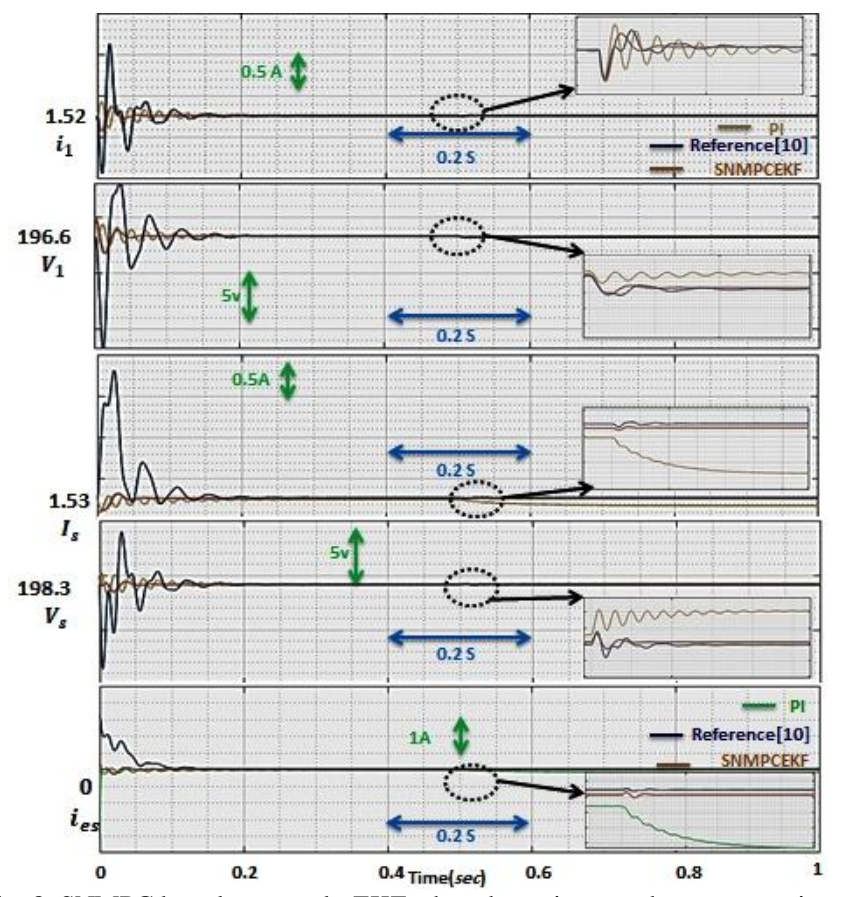

Fig. 8. SNMPC based on pseudo-EKF when the resistance changes over time (Real-time simulation).

To evaluate the performance of the proposed method during the sudden change in the voltage reference, the voltage references are changed as follows:

$$
V_{s}=\left\{\begin{array}{cc}
198.4 & 0<t \leq 0.5 \\
178.2 & 0.5<t \leq 0.7
\end{array}\right.
$$

and the attained results are depicted in Figure 12.

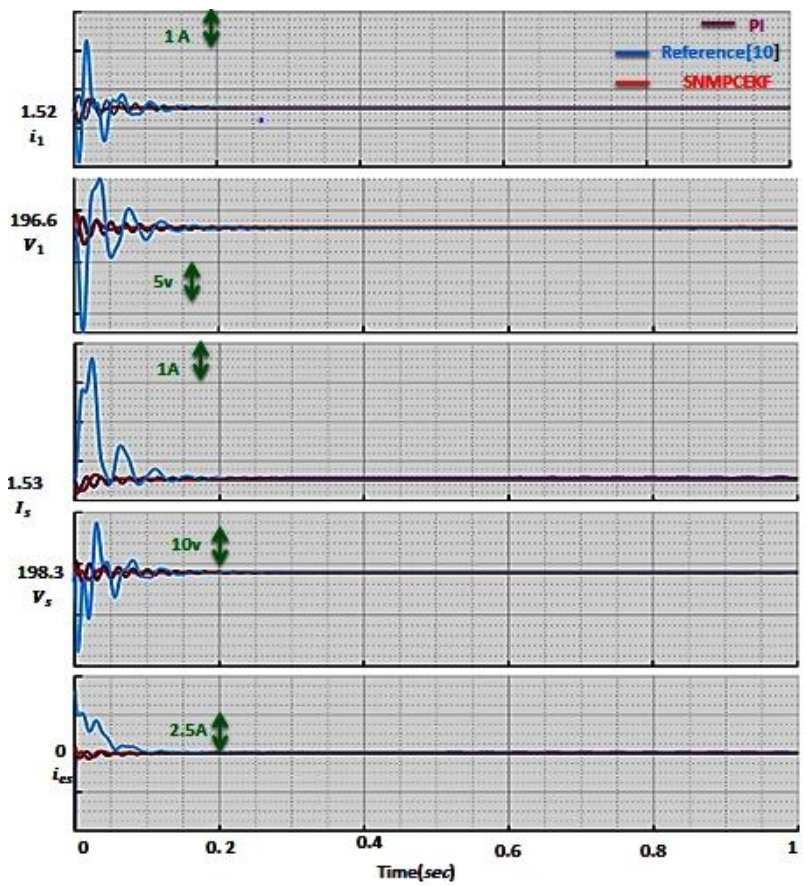

Fig. 9. SNMPC based on the pseudo-EKF when the capacitance changes over time (Real-time simulation). 
To show the advantages of the proposed method, it is compared with two state-of-the-art methods based on the settling time of the systems and their results are presented in Table III. As shown in this table, when the power is changed at time $0.5 \mathrm{~s}$, the proposed method is the fastest method that converges with the operating points and tracks the new operating point with the lowest oscillations.

Therefore, by these tests, the main concerns about the applicability of the proposed method on real hardware are addressed. In other words, it has been shown that:

- the proposed architecture is suitable for the real-world application,

- the proposed method can be implemented on real hardware because the link speeds are adequate.

Table III. The settling time of the system states based on criterion $2 \%$

\begin{tabular}{cccc}
\hline & SNMPCEKF & Reference[10] & PI \\
\hline $\boldsymbol{I}_{\mathbf{1}}$ & $0.397 \mathrm{~ms}$ & $1.587 \mathrm{~ms}$ & $1.846 \mathrm{~ms}$ \\
$\boldsymbol{V}_{\mathbf{1}}$ & $0.38 \mathrm{~ms}$ & $1.475 \mathrm{~ms}$ & $1.765 \mathrm{~ms}$ \\
$\boldsymbol{I}_{\boldsymbol{s}}$ & $0.407 \mathrm{~ms}$ & $1.398 \mathrm{~ms}$ & $1.618 \mathrm{~ms}$ \\
$\boldsymbol{V}_{\boldsymbol{s}}$ & $0.398 \mathrm{~ms}$ & $1.485 \mathrm{~ms}$ & $1.505 \mathrm{~ms}$ \\
\hline
\end{tabular}
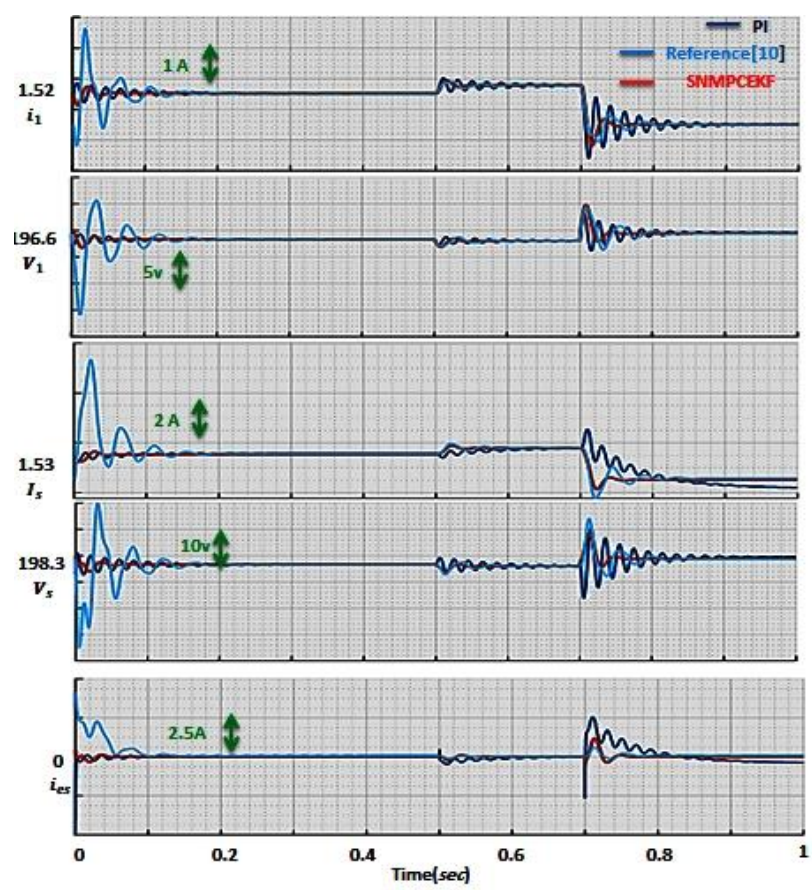

Fig. 10. SNMPC based on the pseudo-EKF when the power changes over time (Real-time simulation).

\section{CONCLUSION}

This paper proposed a novel constrained nonlinear stochastic controller for DC islanded modern power grids with CPLs. Theorem 1 and its proof illustrate the global asymptotic stability of the proposed controller. To examine the practicability of the proposed controller, MiL real-time simulations based on dSPACE were implemented. To this end, the performance of the proposed controller is evaluated in three scenarios. Firstly, to assess the effectiveness of the proposed method, it was compared to the conventional PI, which is a common controller for stabilizing DCMGs and state-of-the-art controllers. The attained results showed that the proposed controller was associated with low oscillations. Thus, the findings showed the performance and advantages of the proposed method in the control of the stand-alone DCMG. Secondly, it was assumed that the resistance of the CPL changed during the test, and the obtained results showed the robustness of the proposed method. Finally, it was assumed that the power of the CPL changes over time, and the findings illustrated that the proposed method can effectively track these variations. The obtained results confirm that the interactions between the controller and the system comply with the system requirements, and verify the superiority of the proposed controller over the state-of-the-art approach.

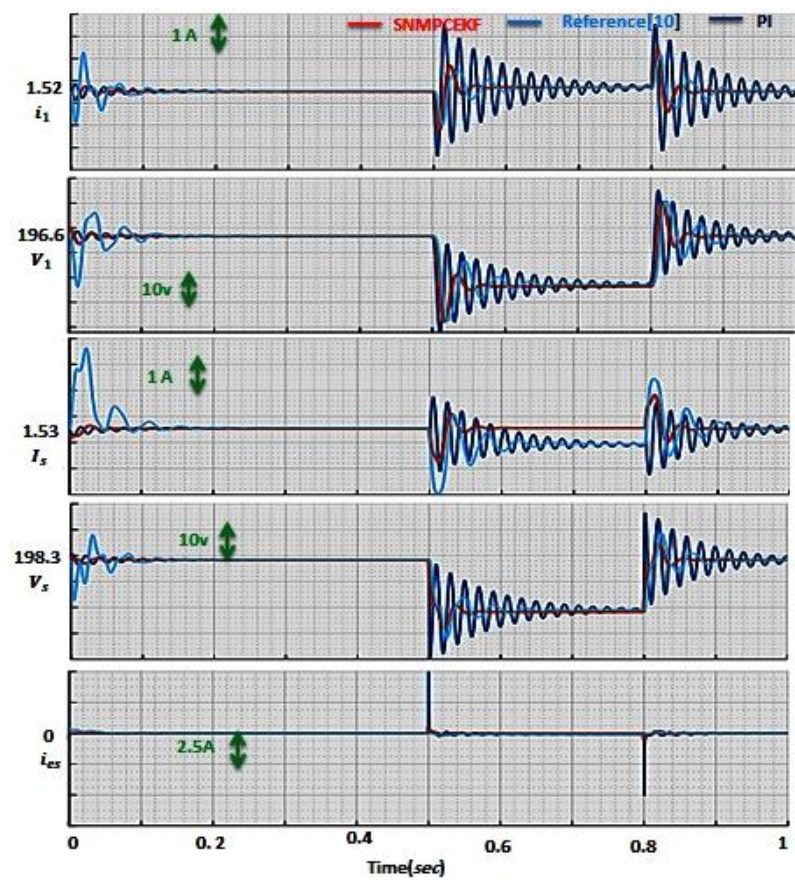

Fig. 11. SNMPC based on the pseudo-EKF when the input voltage changes over time (Real-time simulation).

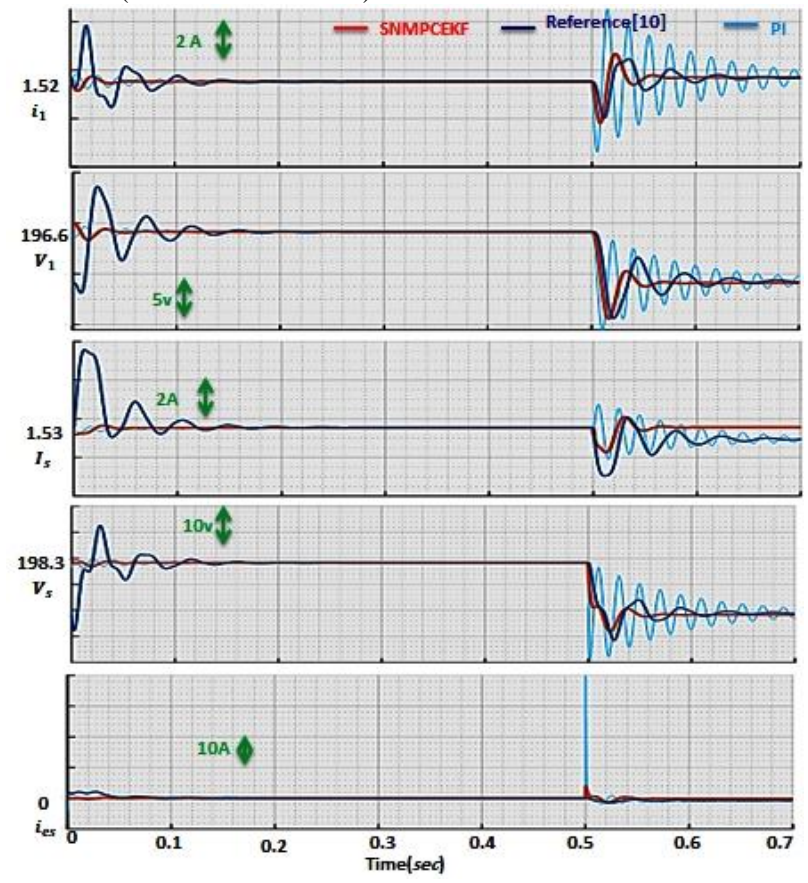

Fig. 12. SNMPC based on the pseudo-EKF when the sudden change occurs in the voltage reference (Real-time simulation). 


\section{REFERENCES}

[1] M.-H. Khooban, "Secondary Load Frequency Control of Time-Delay Stand-Alone Microgrids With Electric Vehicles," IEEE Trans. Ind. Electron., vol. 65, no. 9, pp. 7416-7422, Sep. 2018.

[2] M. Dehghani, M. H. Khooban, T. Niknam, and S. M. R. Rafiei, "Time-Varying Sliding Mode Control Strategy for Multibus LowVoltage Microgrids with Parallel Connected Renewable Power Sources in Islanding Mode," J. Energy Eng., vol. 142, no. 4, p. 05016002, Dec. 2016

[3] M. Gheisarnejad, M.-H. Khooban, and T. Dragicevic, "The Future 5G Network Based Secondary Load Frequency Control in Maritime Microgrids," IEEE J. Emerg. Sel. Top. Power Electron., pp. 1-1, 2019.

[4] M.-H. Khooban, T. Dragicevic, F. Blaabjerg, and M. Delimar, "Shipboard Microgrids: A Novel Approach to Load Frequency Control," IEEE Trans. Sustain. Energy, vol. 9, no. 2, pp. 843-852, Apr. 2018.

[5] K. Lai and M. S. Illindala, "A distributed energy management strategy for resilient shipboard power system," Appl. Energy, vol. 228, pp. 821-832, Oct. 2018

[6] S. K. Sahoo, A. K. Sinha, and N. K. Kishore, "Control Techniques in AC, DC, and Hybrid AC-DC Microgrid: A Review," IEEE J. Emerg. Sel. Top. Power Electron., vol. 6, no. 2, pp. 738-759, Jun. 2018.

[7] A. Kwasinski and C. N. Onwuchekwa, "Dynamic behavior and stabilization of DC microgrids with instantaneous constant-power loads," IEEE Trans. Power Electron., vol. 26, no. 3, pp. 822-834, 2011.

[8] T. Dragičević, X. Lu, J. C. Vasquez, and J. M. Guerrero, "DC microgrids-Part I: A review of control strategies and stabilization techniques," IEEE Trans. Power Electron., vol. 31, no. 7, pp. 48764891, 2015.

[9] T. Dragičević, X. Lu, J. C. Vasquez, and J. M. Guerrero, "DC microgrids-Part II: A review of power architectures, applications, and standardization issues," IEEE Trans. Power Electron., vol. 31, no. 5, pp. 3528-3549, 2015.

[10] M. A. Kardan et al., "Improved stabilization of nonlinear DC microgrids: Cubature Kalman filter approach," IEEE Trans. Ind. Appl., vol. 54, no. 5, pp. 5104-5112, 2018.

[11] N. Vafamand, M. H. Khooban, T. Dragicevic, F. Blaabjerg, and J. Boudjadar, "Robust non-fragile Fuzzy Control of uncertain DC Microgrids Feeding Constant Power Loads," IEEE Trans. Power Electron., pp. 1-1, 2019.

[12] M. M. Mardani, N. Vafamand, M. H. Khooban, T. Dragičević, and F. Blaabjerg, "Design of quadratic D-stable fuzzy controller for DC microgrids with multiple CPLs," IEEE Trans. Ind. Electron., vol. 66, no. 6, pp. 4805-4812, 2018.

[13] T. Dragičević, "Dynamic stabilization of DC microgrids with predictive control of point-of-load converters," IEEE Trans. Power Electron., vol. 33, no. 12, pp. 10872-10884, 2018.

[14] M. M. Mardani, N. Vafamand, M. H. Khooban, T. Dragicevic, and F. Blaabjerg, "Design of Quadratic D-Stable Fuzzy Controller for DC Microgrids With Multiple CPLs," IEEE Trans. Ind. Electron., vol. 66, no. 6, pp. 4805-4812, Jun. 2019

[15] N. Vafamand, S. Yousefizadeh, M. H. Khooban, J. D. Bendtsen, and T. Dragicevic, "Adaptive TS Fuzzy-Based MPC for DC Microgrids With Dynamic CPLs: Nonlinear Power Observer Approach," IEEE Syst. J., pp. 1-8, 2018

[16] G. Sulligoi, D. Bosich, G. Giadrossi, L. Zhu, M. Cupelli, and A. Monti, "Multiconverter medium voltage DC power systems on ships: Constant-power loads instability solution using linearization via state feedback control," IEEE Trans. Smart Grid, vol. 5, no. 5, pp. 2543 2552, 2014.

[17] L. Ding, Q.-L. Han, L. Y. Wang, and E. Sindi, "Distributed Cooperative Optimal Control of DC Microgrids With Communication Delays," IEEE Trans. Ind. Inform., vol. 14, no. 9, pp. 3924-3935, Sep. 2018.

[18] A. M. Rahimi, G. A. Williamson, and A. Emadi, "Loop-cancellation technique: A novel nonlinear feedback to overcome the destabilizing effect of constant-power loads," IEEE Trans. Veh. Technol., vol. 59, no. 2, pp. 650-661, 2010.

[19] E. Hossain, R. Perez, S. Padmanaban, and P. Siano, "Investigation on the development of a sliding mode controller for constant power loads in microgrids," Energies, vol. 10, no. 8, p. 1086, 2017.
[20] Y. Zhao, W. Qiao, and D. Ha, "A sliding-mode duty-ratio controller for DC/DC buck converters with constant power loads," IEEE Trans. Ind. Appl., vol. 50, no. 2, pp. 1448-1458, 2014.

[21] D. Mayne, "Robust and stochastic model predictive control: Are we going in the right direction?," Annu. Rev. Control, vol. 41, pp. 184$192,2016$.

[22] R. O. Lopes, E. M. Mendes, L. A. Torres, and R. Palhares, "Constrained robust model predicted control of discrete-time Markov jump linear systems," IET Control Theory Appl., vol. 13, no. 4, pp. 517-525, 2019.

[23] R. Zhang, A. Xue, and F. Gao, "Model Predictive Control Under Constraints," in Model Predictive Control, Springer, 2019, pp. 59-63.

[24] T. Dragičević, "Model predictive control of power converters for robust and fast operation of ac microgrids," IEEE Trans. Power Electron., vol. 33, no. 7, pp. 6304-6317, 2017.

[25] L. Herrera, W. Zhang, and J. Wang, "Stability analysis and controller design of DC microgrids with constant power loads," IEEE Trans. Smart Grid, vol. 8, no. 2, pp. 881-888, 2015.

[26] X. Xing, H. Meng, L. Xie, L. Yue, and Z. Lin, "Switching Performance Improvement Based on Model-Predictive Control for Wind Turbine Covering the Whole Wind Speed Range," IEEE Trans. Sustain. Energy, vol. 10, no. 1, pp. 290-300, 2019.

[27] M. R. Nasiri, S. Farhangi, and J. Rodríguez, "Model predictive control of a multilevel CHB STATCOM in wind farm application using diophantine equations," IEEE Trans. Ind. Electron., vol. 66, no. 2, pp. 1213-1223, 2019.

[28] L. Asselborn, Control of Stochastic Hybrid Systems based on Probabilistic Reachable Set Computation. kassel university press $\mathrm{GmbH}, 2018$.

[29] W. Yan, B. Zhang, G. Zhao, S. Tang, G. Niu, and X. Wang, "A Battery Management System With a Lebesgue-Sampling-Based Extended Kalman Filter," IEEE Trans. Ind. Electron., vol. 66, no. 4, pp. 3227-3236, 2019.

[30] Y. Chen, H. Li, Z. Qiu, T. Wang, and K. R. Oldham, "Improved Extended Kalman Filter Estimation using Threshold Signal Detection with a MEMS Electrostatic Micro-Scanner," IEEE Trans. Ind. Electron., 2019.

[31] A. Barrau and S. Bonnabel, "The invariant extended Kalman filter as a stable observer," IEEE Trans. Autom. Control, vol. 62, no. 4, pp. 1797-1812, 2016.

[32] J. Zarei, E. Kowsari, and R. Razavi-Far, "Induction Motors Fault Detection Using Square-Root Transformed Cubature Quadrature Kalman Filter," IEEE Trans. Energy Convers., vol. 34, no. 2, pp. 870877, 2018.

[33] J. Zarei, E. Kowsari, and R. Razavi-Far, "Induction Motors Fault Detection Using Square-Root Transformed Cubature Quadrature Kalman Filter," IEEE Trans. Energy Convers., vol. 34, no. 2, pp. 870877, Jun. 2019.

[34] D. Simon, Optimal state estimation: Kalman, $H$ infinity, and nonlinear approaches. John Wiley \& Sons, 2006.

[35] S. Yousefizadeh, J. D. Bendtsen, N. Vafamand, M. H. Khooban, F. Blaabjerg, and T. Dragicevic, "Tracking Control for a DC Microgrid Feeding Uncertain Loads in More Electric Aircraft: Adaptive Backstepping Approach," IEEE Trans. Ind. Electron., vol. 66, no. 7, pp. 5644-5652, Jul. 2019.

[36] N. Vafamand, M. H. Khooban, T. Dragicevic, and F. Blaabjerg, "Networked Fuzzy Predictive Control of Power Buffers for Dynamic Stabilization of DC Microgrids," IEEE Trans. Ind. Electron., vol. 66, no. 2, pp. 1356-1362, Feb. 2019.

[37] S. Yousefizadeh, J. D. Bendtsen, N. Vafamand, M. H. Khooban, T. Dragicevic, and F. Blaabjerg, "EKF-Based Predictive Stabilization of Shipboard DC Microgrids With Uncertain Time-Varying Load," IEEE J. Emerg. Sel. Top. Power Electron., vol. 7, no. 2, pp. 901-909, Jun. 2019.

[38] M. M. Mardani, N. Vafamand, M. H. Khooban, T. Dragicevic, and F. Blaabjerg, "Design of Quadratic D-Stable Fuzzy Controller for DC Microgrids With Multiple CPLs," IEEE Trans. Ind. Electron., vol. 66, no. 6, pp. 4805-4812, Jun. 2019.

[39] J. A. Andersson, J. Gillis, G. Horn, J. B. Rawlings, and M. Diehl, "CasADi: a software framework for nonlinear optimization and optimal control," Math. Program. Comput., vol. 11, no. 1, pp. 1-36, 2019. 